\title{
Regulation of the Circadian Clock in the Aplysia Eye: Mimicry of Neural Action by Serotonin
}

\author{
John J. Nadakavukaren, ${ }^{1}$ Marvin E. Lickey, and Wesley P. Jordan ${ }^{2}$ \\ Departments of Biology and Psychology and Institute of Neuroscience, University of Oregon, \\ Eugene, Oregon 97403
}

In Aplysia, activity of efferent fibers in the optic nerve can influence the circadian clock in the eye. In addition, serotonin (5HT) is present in the eye and influences the function of the clock. Accordingly, we hypothesized that 5-H'T is the transmitter of the optic efferents, and tested the prediction that exogenous 5-HT would mimic the action of the optic efferents on the clock. We also tested the prediction that the action of the efferents would be prevented by blocking synaptic secretions with high $\mathrm{Mg}^{2+}$, low $\mathrm{Ca}^{2+}$ (HMLC). Activity of the optic efferents enhances clock resetting in response to the onset of darkness. We used this neural enhancement as a measure of neural action on the eye clock.

We found that (1) HMLC blocked neural enhancement. (2) Serotonin enhanced resetting to the same extent as efferent activity. (3) Enhancement by 5-HT did not summate with neural enhancement. (4) High concentrations of 5-HT ([5-HT] $\geq 6 \times$ $10^{-4}$ ) did not cause enhancement. (5) High concentrations of 5-HT also blocked neural enhancement. (6) Enhancement by 5-HT depended on the phase of drug application, much as neural enhancement depends on the phase of neural activity. (7) Enhancement by 5-HT depended on day length in a manner similar to the enhancement by neural activity. (8) HMLC did not block the facilitatory action of 5-HT. (9) Several other transmitters that may be active in the eye did not enhance resetting.

We conclude that a secretory step is required for neural enhancement of resetting and that bath-applied 5-HT parametrically mimics the effects produced by activity of the optic efferent axons. This result greatly strengthens the hypothesis that 5-HT is a clock-regulatory transmitter of the optic efferent axons in Aplysia. This is the first time that a putative neurotransmitter has been shown to mimic a neural action on a circadian clock.

There are several indications that the circadian clock in the Aplysia eye is regulated by the activity of serotonergic efferent axons in the optic nerve. The efferent fibers were first described by Eskin (1971), and in several experimental paradigms, the action of light on the phase of the eye clock is different when efferent activity is present than when it is absent (Block et al., 1974; Block and Page, 1979; Prichard and Lickey, 1981a, b).

\footnotetext{
Received Nov. 19, 1984; revised July 18, 1985; accepted July 19, 1985.

We thank Robert G. Prichard for assistance and discussion. A major part of this work appeared in the doctoral dissertation of J.N., University of Oregon (1980). This research was supported by NSF Grants 28251 and 08168 to M.E.L.

Correspondence should be sent to Marvin E. Lickey, Institute of Neuroscience, University of Oregon, Eugene, OR 97403

' Current address: Department of Pharmacology, Dana-Farber Cancer Institute, Harvard Medical School, Boston, MA 02115.

${ }^{2}$ Current address: Division of Human Development, St. Mary's College of Maryland, St. Mary's City, MD 20686.

Copyright (C) 1986 Society for Neuroscience $0270-6474 / 86 / 010014-08 \$ 02.00 / 0$
}

Evidence that the optic efferents may be serotonergic includes the findings that (1) pulse doses of exogenous 5-HT can reset the eye clock in vitro, (2) 5-HT is present in the eye, and (3) $5-\mathrm{HT}$ is synthesized in the eye (Corrent et al., 1978, 1982).

This paper reports experiments to test the hypothesis that exogenous 5-HT mimics the action of the optic efferents on the eye clock. If 5-HT is, indeed, the transmitter of the optic efferents, then efferent neural activity and exogenous 5-HT should affect the eye clock in the same way.

The clearest demonstration that efferent neural activity influences the eye clock was provided by Prichard and Lickey (1981a $b, c)$. They found that the response of the eye clock to the onset of darkness depends on activity of the efferent axons. Under appropriate experimental circumstances, the onset of darkness produces only small resets when efferent activity is absent, but produces large resets when efferent activity is present. We call this phenomenon "neural enhancement of resetting."

Neural enhancement of resetting has several properties that make it useful for testing the hypothesis that 5-HT mimics the action of the optic efferents. First, neural enhancement occurs in vitro, where it is possible to apply exogenous 5-HT and other drugs in a controlled manner. Second, the effective efferent activity occurs at a particular phase of the rhythm, 3-6 hr after projected dawn. Third, the magnitude of the neurally enhanced reset is determined by the duration of light on the final day preceding the onset of darkness. For example, if the duration of light is less than $12 \mathrm{hr}$, dark onset does not reset the eye clock at all. If the duration of light is $18 \mathrm{hr}$, dark onset resets an enervated eye by about $6 \mathrm{hr}$ but resets a denervated eye hardly at all. If duration of light is $21 \mathrm{hr}$, dark onset resets an enervated eye by about $11 \mathrm{hr}$, but a denervated eye by only about $3 \mathrm{hr}$.

In the present studies, we tested whether bath application of $5-\mathrm{HT}$ enhances resetting in the same way that efferent neural activity does. We found that 5-HT does mimic the action of the optic efferents. This is the first time that a putative transmitter has been shown to mimic a nerve action on an identified circadian clock.

\section{Materials and Methods}

We recorded the rhythm of compound action potentials (CAPs) from the two eyes of the same Aplysia while maintaining one eye in artificial seawater (ASW) and giving the other eye a drug pulse. The standard experimental design is illustrated in Figure $1 A$. The animals were exposed to light cycles of $12 \mathrm{hr}$ of light and $12 \mathrm{hr}$ of dark (LD 12/12) for 3 or more days after they arrived in the laboratory from southern California. The light source was fluorescent, and the intensity was about $500 \mathrm{~lx}$ at the surface of the water. During the light portion of the last light cycle before a run, two animals were transferred to a different tank, in which the duration of light was prolonged beyond the usual $12 \mathrm{hr}$. In most cases, the duration of light was $21 \mathrm{hr}$. Just before the end of the extended light, the eyes and cerebral ganglion were removed from 
A
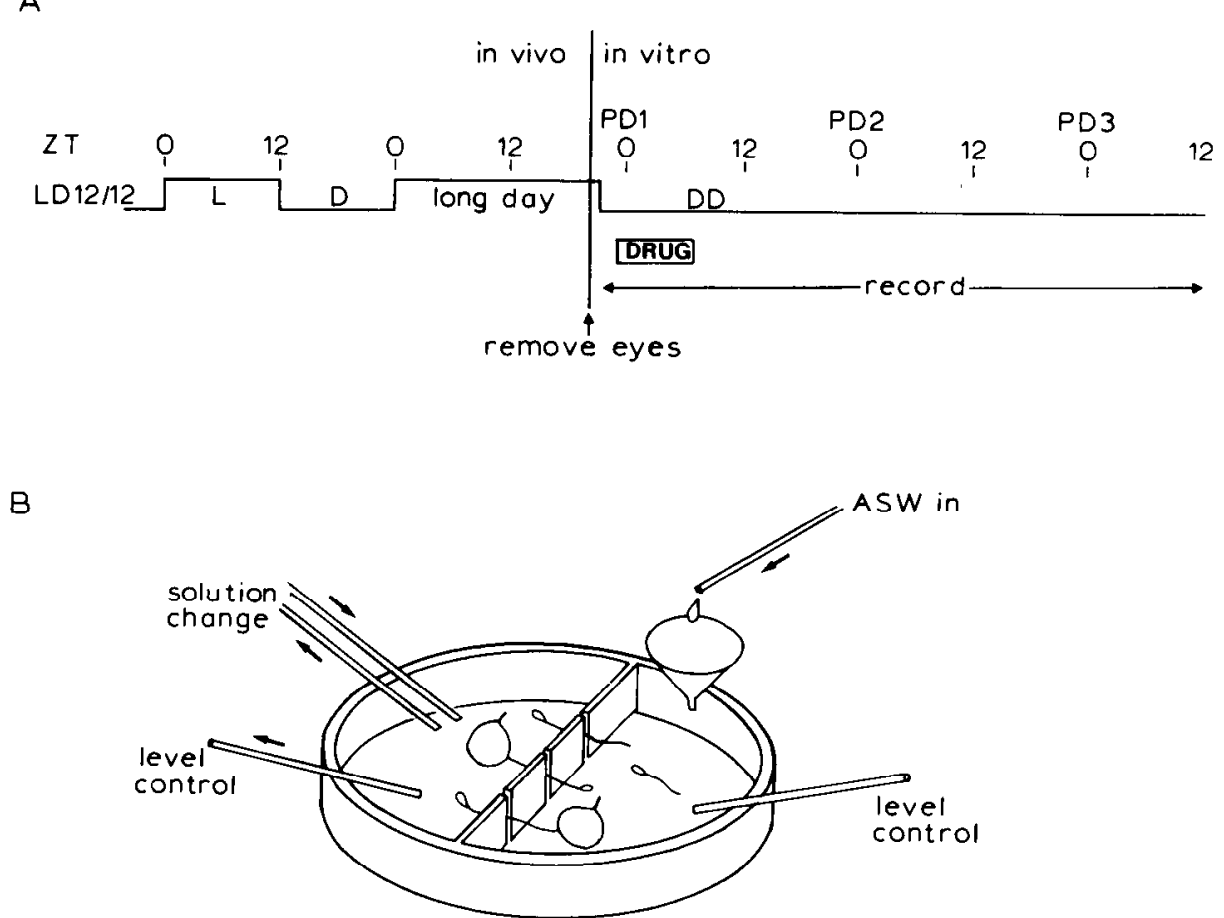

Figure 1. A, Experimental design. Horizontal axis: Zeitgeber time. In vivo light cycles indicated by line that is up during lighttime, down during darktime. Eyes and cerebral ganglion were removed for recording during the final half hour of the long day; one optic nerve was cut and the other remained intact. Dark onset occurred a few minutes after the nerve cut when the preparation was darkened for recording. In the standard procedure, drugs were applied at ZT 23-7. The second optic nerve was cut at the end of the drug pulse, at ZT 7. PD1, PD2, PD3: Projected dawns 1, 2, and 3. Data were typically obtained between PD1 and PD2. B, Recording chamber with two compartments. Sperm-shaped objects represent eyes with attached optic nerve. Circular objects represent cerebral ganglia. The experimental setup illustrates the conditions that applied during the doseresponse experiments (Fig. 5). Three of the optic nerves are shown threaded through slits in the partition between the compartments. Drugs were applied on the left side only via the tubes marked solution change. The non-drug (right) side was continuously perfused with fresh ASW. Suction was applied to level control tubes to prevent overflow. Suction electrodes (not shown) were applied to nerves on right side only. One of the drugtreated eyes is attached to the untreated cerebral ganglion. The companion treated eye is detached. On the left is an untreated detached eye and nerve from a second animal. The companion for this eye was attached to its ganglion, and the ganglion received drug treatment. The results from these ganglion-treated attached eyes are not reported here (see Nadakavukaren, 1980).

both animals for drug treatment and recording in vitro. In some experiments (Figs. 2 and 7), the duration of light on the final day was manipulated as an independent variable.

The two pairs of eyes, together with the cerebral ganglia, were placed in a Plexiglas chamber (Fig. $1 B$ ). The chamber had two semicircular compartments separated by a partition. The volume of each compartment was about $90 \mathrm{ml}$. One compartment was for drug-treated eyes, and the other was for control eyes in ASW. Three $0.35 \mathrm{~mm}$ wide slits were cut in the wall. The thickness of the wall at the slits was less than $1 \mathrm{~mm}$. Optic nerves were threaded through the slits, and the slits were sealed with petroleum jelly so that the two chambers were pharmacologically isolated. The control compartment was continuously perfused with fresh ASW to insure that drug concentration could not build up in the control compartment via leakage through the seal. One of the eyes from each animal was isolated from the cerebral ganglion by cutting its optic nerve. Suction electrodes were then applied to all optic nerves. The preparation was then darkened, terminating the extended day. This darkening was the resetting stimulus. In the standard procedure (as in the dose-response experiment, Fig. 5), the nerve of the attached eye was also cut $10 \mathrm{hr}$ after dark onset at ZT 7 (ZT = zeitgeber time; ZT $0=$ the time at which the lights came on in the LD $12 / 12$ pretreatment). Thus, the difference between an attached eye and a detached eye was that the attached eye received input from the cerebral ganglion via the optic efferents for $10 \mathrm{hr}$ after dark onset (ZT 21-7), while the detached eye received no neural input after dark onset. The preparation received a 1-5-min light pulse at the time the nerve of the attached eye was cut. Such a brief pulse has no effect on the subsequent phase of the eyes (Prichard and Lickey, 1981a).

In the standard procedure (Fig. 1 $A$ ), drugs were applied during the early projected day of the first cycle in vitro. Drug onset was just before projected dawn at ZT 23; drug offset was at just after projected midday at ZT 7. The drug treatment terminated at the same time that the nerve of the attached eye was severed. Thus, drug treatment occurred at the same time that attached eyes received neural inputs from the cerebral ganglion. This timing allowed direct comparison between the eye's response to drugs and the eye's response to efferent neural activity. Prichard and Lickey $(198 \mathrm{lb}$ ) showed that early subjective day is a time when neural activity is especially potent in modulating resetting. In one experiment (Fig. 6), the time of drug application was a manipulated variable, and in some experiments both eyes were detached (Figs. 6 and 7).

The ASW contained the following concentrations of salts (mм): $[\mathrm{NaCl}]=425,[\mathrm{KCl}]=10,\left[\mathrm{CaCl}_{2}\right]=10,\left[\mathrm{MgCl}_{2}\right]=22,\left[\mathrm{MgSO}_{4}\right]=26$, and $[\mathrm{HEPES}]=10$. High-magnesium, low-calcium ASW contained (in mм) $[\mathrm{NaCl}]=320,[\mathrm{KCl}]=10,\left[\mathrm{CaCl}_{2}\right]=0.1$ or $1.0,\left[\mathrm{MgCl}_{2}\right]=125$, $\left[\mathrm{Na}_{2} \mathrm{SO}_{4}\right]=28$, and [HEPES] - 10. All solutions had a pH of 7.9-8.0 and contained 10,000 units of penicillin and $10,000 \mathrm{mcg}$ streptomycin/ $100 \mathrm{ml}$ of working solution. Solutions of 5-HT, carbamylcholine, and octopamine were made by adding the drug directly to the ASW. Apomorphine was first dissolved in double-distilled water to make a $1 \mathrm{~mm}$ stock solution; an appropriate amount of this solution was then added to the ASW. Dopamine was dissolved in ASW containing $1 \mathrm{mM} \mathrm{L}-$ ascorbate before being added to the recording compartment. Solutions in the compartments were changed without illuminating the preparation. The temperature of the preparation was $15^{\circ} \mathrm{C}$, and there were no thermal shocks when solutions were changed. A solution change consisted of three washings with the new medium, followed by a filling.

The measured variable was the phase of the CAP rhythm. Phase was defined as the time of the midpoint of the rising phase. Time was scaled in hours since projected dawn (ZT). Most of the data were from the first cycle after drug application. On some occasions, when a malfunction obscured pertinent observations, data were taken from subsequent cycles. Nearly all the rhythms were observed for more than one cycle beyond drug application, and the phase of later cycles was not materially different from the phase of the first. 


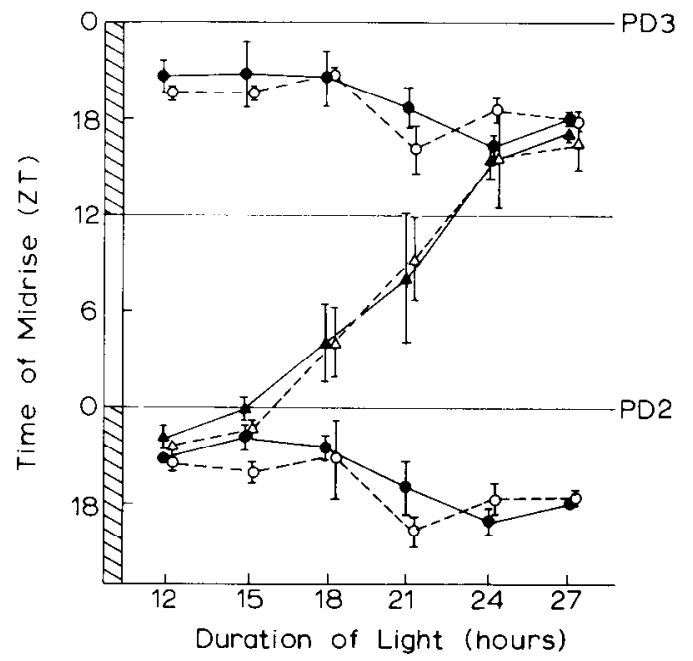

Figure 2. Neural enhancement of resetting. Abscissa: Duration of light on final long day. Ordinate: Zeitgeber time of midrise point of eye rhythms, mean \pm SD. Rectangles on ordinate show projected night (hatched) and projected day (open). Solid symbols and solid lines show results of Prichard and Lickey (1981a). Open symbols and dashed lines show recent replication of Prichard and Lickey. Circles: Detached eyes. Triangles: Attached eyes. Points slightly displaced horizontally avoid overlap. PD2, PD3: Second and third projected dawns. Three cycles were recorded for all eyes, but not all cycles are shown. The first two cycles are shown for detached eyes; only the first cycle is shown for attached eyes. Note: When duration of light is $15-24 \mathrm{hr}$, the attached eyes have a different phase than the detached eyes, especially when duration of light is $21 \mathrm{hr}$. Pooling the data from all cycles and both replications, the phase difference between the attached and detached eyes is statistically significant ( $t$ test for correlated means) at the $5 \%$ level or better for light durations of $15 \mathrm{hr}(p<0.02), 18 \mathrm{hr}(p<0.01)$, $21 \mathrm{hr}(p<0.01)$, and $24 \mathrm{hr}(p<0.05)$.

\section{Results}

\section{Background}

Neurally enhanced resetting is illustrated in Figure 2 by data from Prichard and Lickey (1981a) and by a recent replication. The duration of the last day of LD was varied over the range of 9-30 hr. Within this range, the offset of light caused a different degree of resetting in attached eyes than in detached eyes.

The standard phase of the eye rhythm may be defined as the phase obtained when the duration of light on the final day of LD is $12 \mathrm{hr}$. This condition is identical to the conventional practice of recording from detached eyes after in vivo entrainment to LD 12/12. In agreement with much previous work (e.g., Block et al., 1974; Corrent et al., 1982; Eskin, 1971; Jacklet, $1969,1974)$, the midrise point under these standard conditions occurred a few hours before projected dawn (Fig. 2).

When we lengthened the duration of light beyond $12 \mathrm{hr}$ by postponing dark onset, the phase of attached eyes was affected to a greater extent than the phase of detached eyes (Fig. 2). Attached cycs were markedly reset from their standard phase when the duration of light was 18 or $21 \mathrm{hr}$. By contrast, detached eyes were reset by a much smaller amount, if at all. This neural enhancement of resetting could produce a $12 \mathrm{hr}$ phase difference between attached and detached eyes when the duration of light was near $21 \mathrm{hr}$.

\section{High magnesium, low calcium ASW blocks neural enhancement of resetting}

Neural enhancement of resetting was blocked by bathing attached eyes in HMLC ASW during the period of attachment (Fig. 3). An initial baseline experiment replicated the observation that a $21 \mathrm{hr}$ day produces a larger reset in attached eyes

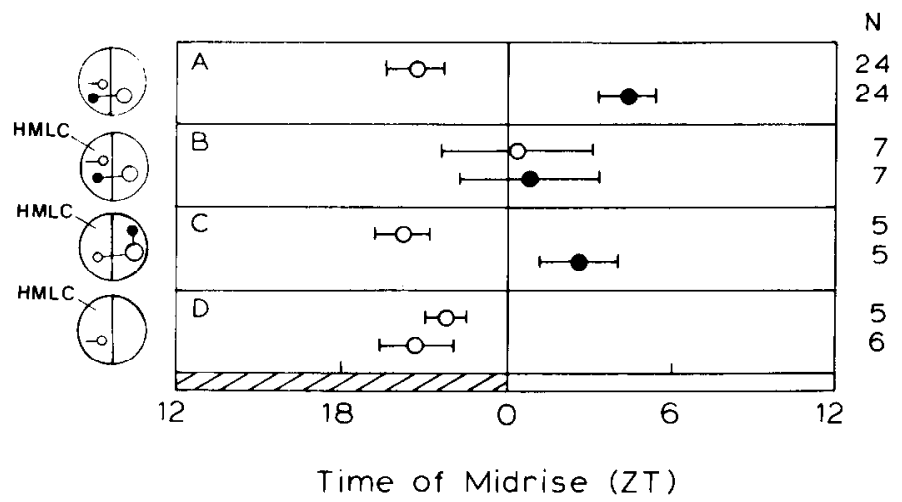

Figure 3. Effect of HMLC on neural enhancement of resetting. Horizontal axis: Phase of eye rhythms, mean \pm 2 SEM. Duration of light, $21 \mathrm{hr}$. Open and filled circles defined by legend on left, which depicts the two-compartment recording chamber. The large circles represent cerebral ganglia, the small circles represent eyes, and the lines represent optic nerves. $A$, Normal phase of untreated attached and untreated detached eyes. $B$, In the presence of HMLC there is no difference between the attached and detached eyes. The phase of these eyes, however, is later and more variable than in normal detached untreated eyes. $C$, HMLC blocks the effect of attachment. $D$, Confirmation that HMLC, itself, does not cause phase shifts (cf. detached eyes in $A$ ). The companion eyes (not shown) were given treatments other than attachment or HMLC (see text).

than in detached eyes (Fig. $3 A$ ). In contrast, there was no difference in the resetting behavior of attached and detached eyes when both were bathed in HMLC solution during the period of attachment (Fig. 3B). Surprisingly, in this experiment the phase was unusually variable and was about $4 \mathrm{hr}$ later than usual for detached eyes in normal ASW (see below). In a third experiment (Fig. 3C), application of HMLC to one member of a pair of atlached eyes caused a large phase difference to develop. These experiments indicate that HMLC blocks the action of the optic nerve.

The unusual phase and large variability of the eyes in Figure $3 B$ could mean that HMLC itself can cause phase shifts in detached eyes. To check this possibility, we observed two additional samples of detached eyes treated with HMLC (Fig. 3D). In these samples, HMLC had no phase-shifting effect. Other workers concur that HMLC itself does not cause phase shifts (Eskin and Corrent, 1977; Jacklet, 1973). We have no secure explanation for the abnormal phase and variability of the cycs in Figure $3 B$.

The blockage of the neural enhancement of resetting by HMLC is consistent with the interpretation that chemical synapses in the eye are essential for such enhancement. Chemical synapses in the ccrebral ganglion are probably also necessary, as HMLC applied to the ganglion also blocks neural enhancement $(\mathrm{Na}$ dakavukaren, 1980).

\section{Serotonin mimics neural enhancement}

Serotonin induced a dramatic phase shift in detached eyes when the duration of light was $21 \mathrm{hr}$. Figure 4 shows an example. Serotonin at a concentration of $10^{-5} \mathrm{M}$ was present during the $8 \mathrm{hr}$ interval of ZT 23-ZT 7 during the second cycle in vitro. This record was taken from an experiment (Fig. 6) in which the time of drug application was the manipulated variable. As reported by others (Corrent et al., 1978, 1982; Eskin, 1977), when 5-HT was in the chamber, the CAP rate was suppressed. When the 5-HT was removed, activity recovered, and the circadian rhythm reappeared at a new phase.

Dose-response curve. Our first step in testing whether 5-HT mimics neural enhancement of resetting was to search for the most effective dose of 5-HT and to compare its action on at- 


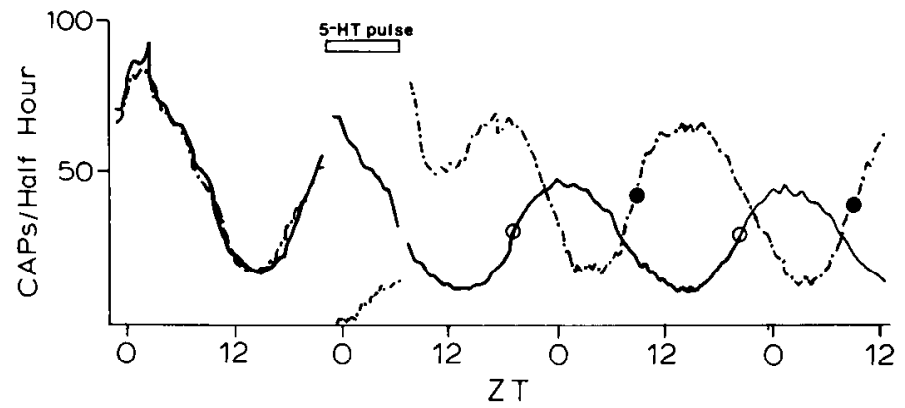

Figure 4. Enhancement of resetting by 5-HT. Duration of light is 21 hr. 5-HT given at ZT 23-7 of second cycle in vitro. Dashed line: Drug treated. Solid line: Untreated. Both eyes detached from the outset. Note the suppression of neural activity during $5-\mathrm{HT}$ treatment and the large reset following the treatment.

tached and detached eyes. If neural efferents and 5-HT activate the same mechanism, then the magnitude of resetting in detached eyes that receive 5-HT should match that of attached eyes that receive no active drug. Also, the effects of 5-HT and efferent activity should not summate in attached eyes. Both of these predictions were borne out (Fig. 5).

At low concentrations $\left(10^{-8}\right.$ and $\left.10^{-7} \mathrm{M}\right), 5-\mathrm{HT}$ produced no phase shifts. Drug-treated detached eyes had the same phase as untreated, detached eyes; drug-treated attached eyes had the same (shifted) phase as untreated attached eyes. At high concentrations $\left(6 \times 10^{-4}\right.$ and $\left.2 \times 10^{-3} \mathrm{M}\right), 5-\mathrm{HT}$ did not shift the phase of detached eyes, and it largely blocked the phase shift that is usually induced by neural activity. At intermediate concentrations $\left(10^{-6}\right.$ and $\left.10^{-5} \mathrm{M}\right), 5-\mathrm{HT}$ induced phase shifts in detached eyes that precisely matched the phase shifts in attached eyes. The phase-shifting action of intermediate concentrations did not summate with the phase-shifting action of the nerve. These facts suggest that $5-\mathrm{HT}$ and nerve activity have the same mechanism of phase-shifting.

The efficacy of 5-HT is phase-dependent. Prichard and Lickey (1981b) found that neural enhancement of resetting was phasedependent. Efferent neural activity confined to the interval of ZT 3-6 of the first cycle in vitro induced phase shifts that were nearly as large as those induced when efferent activity was present at all phases. In contrast, efferent activity confined to the interval, ZT 21-3, caused only a small phase difference between attached and detached eyes.

The phase-shifting action of 5-HT is also phase-dependent (Fig. 6). In contrast to the large phase shifts that resulted when 5-HT was applied at ZT 23-7, 5-HT at ZT 7-15 failed completely to enhance resetting. Serotonin at ZT 15-23 induced phase shifts that were typically smaller than maximal size. Serotonin at ZT 23-7 during the sccond cycle in vitro was as cffective as 5-HT at the same phase during the first cycle. We have not applied 5-HT at times later than ZT 7 of the second cycle in vitro. The $8 \mathrm{hr}$ phase interval during which $5-\mathrm{HT}$ produced the largest phase shifts included the interval during which neural activity produced large phase shifts in the studies of Prichard and Lickey (1981b). A higher-resolution study, using briefer 5HT pulses, might reveal a larger interval during which $5-\mathrm{HT}$ is ineffective.

Size of reset depends on the duration of light. As seen in Figure 2 , the size of the phase shift induced by neural activity is dependent on the duration of light. When we applied $10^{-5}$ M 5-HT to detached eyes, we obtained a similar relationship between duration of light and magnitude of resetting. Figure 7 shows the phase shifts produced by 5-HT following light durations of 12 , $18,21,24$, and $30 \mathrm{hr}$. There were three groups of eyes: (1) detached control, (2) detached and treated with 5-HT, and (3) attached and treated with 5-HT. With a light duration of $12 \mathrm{hr}$,

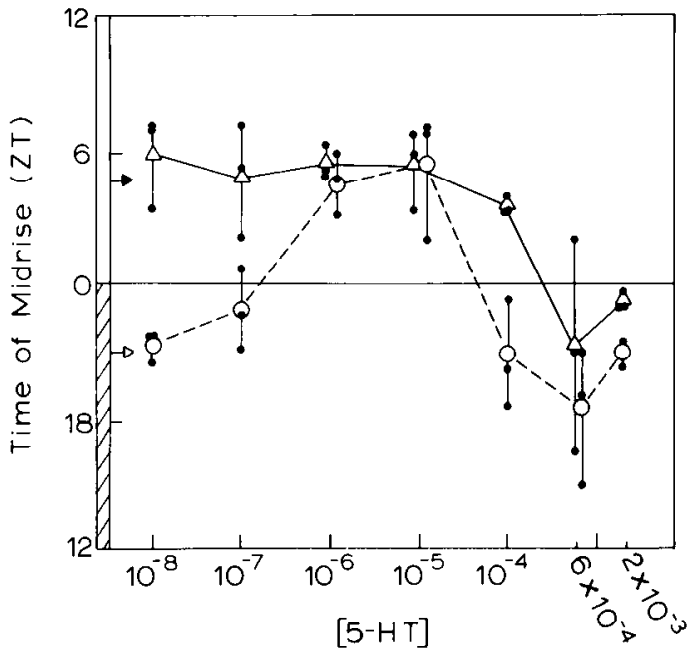

Figure 5. Dose-response curve for enhancement of resetting by 5-HT. Abscissa: 5-HT concentration. Ordinate: Phase of rhythm on first cyclc following 5-HT treatment. Experimental design as in Fig. 1A. Each animal contributed one detached eye and one attached eye. Circles: Mean phase of detached eyes. Triangles: Mean phase of attached eyes. Vertical lines: Range. Dots: Individual eyes. Points slightly displaced horizontally when necessary to avoid overlap. Projected light cycles shown on ordinate. Arrows at left show expected phase for attached (filled arrow) and detached (open arrow) eyes based on data of Fig. $3 A$. For all eyes, the duration of light was $21 \mathrm{hr}$ and $5-\mathrm{HT}$ was applied at ZT $23-7$ of the first cycle in vitro. Note: At $[5-\mathrm{HT}] \geq 6 \times 10^{-4} \mathrm{M}, 5-$ HT does not enhance resetting and blocks neural enhancement. At [5$\mathrm{HT}]=10^{-5} \mathrm{M}, 5-\mathrm{HT}$ mimics neural enhancement but does not summate with it. At $[5-\mathrm{HT}]=10^{-8} \mathrm{M}, 5-\mathrm{HT}$ has no action.

eyes from all three groups had about the same phase. However, when the light duration was 18,21 , or $24 \mathrm{hr}$, attached eyes and detached eyes treated with 5 -HT had a different phase than detached untreated controls. As could be expected from Figure 5 , there was little difference between attached 5-HT-treated eyes and detached 5-HT-treated eyes. The functional relation between the duration of light and the phase of 5-HT-treated eyes was a good match to the corresponding relation obtained by

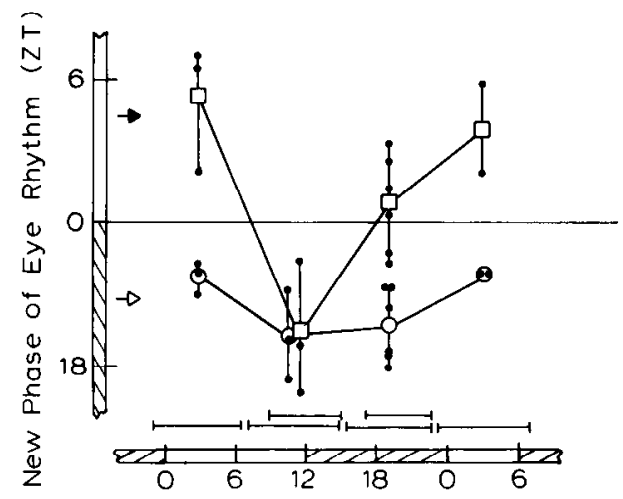

Figure 6. Phase-response curve for action of 5-HT. Abscissa: Phase of 5-HT treatment. Ordinate: New phase of eye rhythms on first cycle after drug treatment. All eyes detached. Circles: Mean phase of untreated detached eyes. Squares: Mean phase of detached eyes treated with $10^{-5}$ M 5-HT. Vertical lines: Range. Dots: Individual data. Points slightly displaced horizontally when necessary to avoid overlap. The time of drug treatment is indicated by lines at bottom. The standard duration of drug treatment was $8 \mathrm{hr}$, but in some preparations the treatment lasted only $6 \mathrm{hr}$. Arrows at left show expected phase for attached (filled arrow) and detached (open arrow) eyes based on data of Fig. 3A. Note: 5-HT pulses beginning in the second half of the projected day do not enhance resetting. 


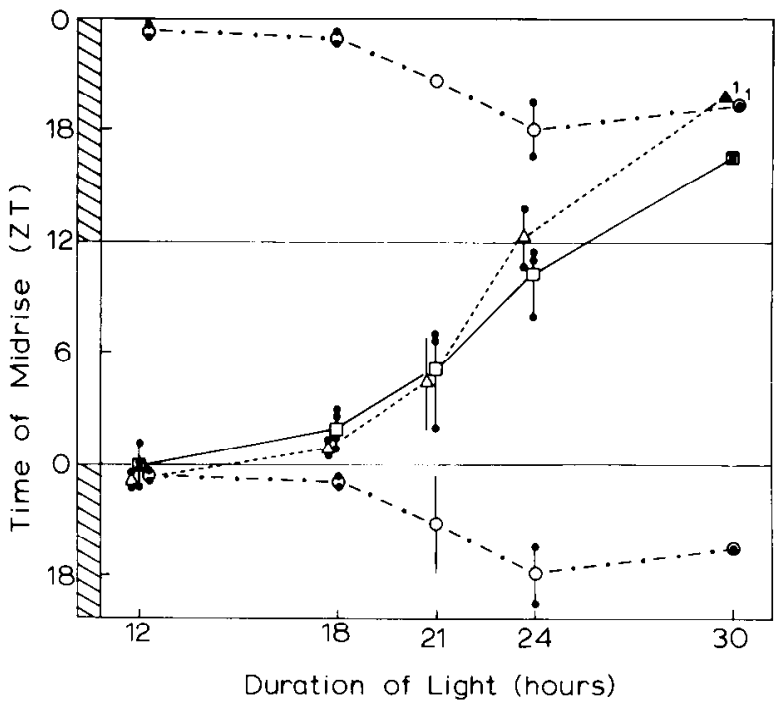

Figure 7. Relation between duration of light and magnitude of enhanced resetting. Abscissa: Duration of light. Ordinate: Mean phase of eye rhythms following 5-HT treatment; two or three cycles after drug treatment are averaged. Squares: 5-HT-treated detached eyes (drugenhanced resetting); 5-HT applied at ZT 23-7 on first cycle in vitro. Triangles: Attached untreated eyes (neurally enhanced resetting), nerve cut at ZT 7. Circles: Detached untreated eyes (no enhancement of resetting). Data from detached eyes (squares) are double-plotted. Projected night (hatched rectangle) and projected day (open rectangle) shown on ordinate. For attached untreated eyes (triangles) and detached untreated eyes (circles), when duration of light is $21 \mathrm{hr}$, the vertical line shows $\pm \mathrm{SD}, N=24$ (from Fig. $3 A$ ); for other points, the vertical lines give range and dots give individual eyes. For two points marked with $s u$ perscript 1 , only one eye per point. Note: Enhancement by 5 -HT mimics enhancement by efferent neural activity (compare squares with triangles). New data for attached and detached eyes are a good match for previous data (cf. Fig. 2).

Prichard and Lickey (1981a) between duration of light and phase of attached untreated eyes (cf. Fig. 2).

\section{Effect of HMLC on resetting action of 5- $H T$}

To discover whether chemical synapses are required to mediate the action of 5-HT, we asked whether HMLC could block the phase shifts produced by 5 -HT. A test was performed according to two protocols. In one protocol, a group of detached eyes was treated with IIMLC plus 5-HT; control eyes from the same animals received 5-HT only. In the second protocol, detached eyes were treated with HMLC plus 5-HT and controls were treated with HMLC only. The results are given in Figure 8.

There was no significant difference between the mean phase shift produced by HMLC plus 5-HT and that produced by 5-HT alone. There was a significant difference between eyes treated with HMLC alone and those treated with 5-HT plus HMLC. The phase of eyes treated with HMLC alone was not different from the normal phase for untreated eyes. Thus, chemical synaptic transmission is probably not involved in mediating the phase-shifting action of 5-HT. The phase of the 5-HT-treated eyes shown in Figure 8 was delayed in comparison to previous experiments (cf. Fig. 7; light duration $=21 \mathrm{hr}$ ). We do not know the cause of this difference. It is our impression that there is greater variability in eyes that have recently experienced a large phase shift than in eyes that have not experienced a shift (e.g., see Fig. 7). The unusually late phase of 5-HT-treated eyes in Figure 8 is probably a manifestation of this greater variability.

\section{Other transmitters do not enhance resetting}

The eye is known to contain dopamine and octopamine, and the neural activity of the retina responds to bath-applied ace-

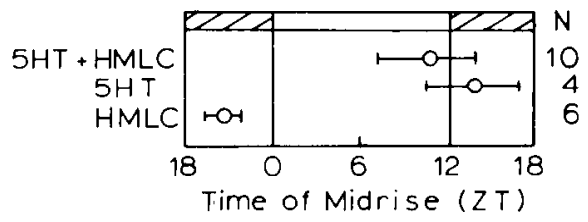

Figure 8. Action of 5-HT in the presence of HMLC. Mean phase \pm 2 SEM on first cycle after drug treatment. Day length $=21 \mathrm{hr}$. Drugs applied at ZT 23-7 of the first cycle in vitro. Note: HMLC does not affect the action of 5-HT, and HMLC alone does not change the phase from the value expected from detached untreated eyes.

tylcholine (Corrent and Eskin, 1982; Corrent et al., 1978; Eskin, 1977; Jacklet, 1968; Luborsky-Moore and Jacklet, 1976). Agonists of these transmitters did not cause phase-shifting when applied at the same time (ZT 23-7) and in the same concentration $\left(10^{-5} \mathrm{M}\right)$ at which $5-\mathrm{HT}$ was effective. Creatinine sulfate, which was complexed with the 5-HT used in this study, did not by itself cause phase-shifting. We have not obtained complete dose response curves for these agents.

\section{Discussion}

\section{Secretory step in neural enhancement of resetting}

Neural enhancement of resetting is blocked by treatment of the eye with artificial seawater having high $\left[\mathrm{Mg}^{2+}\right]$ and low $\left[\mathrm{Ca}^{2+}\right]$. The concentrations of $\mathrm{Mg}^{2+}$ and $\mathrm{Ca}^{2+}$ we used are known to prevent chemical synaptic transmission and the release of secretory products in the Aplysia eye and elsewhere in the Aplysia nervous system (Cedar and Schwartz, 1972; Halstead and Jacklet, 1974; Harf et al., 1976). HMLC does not, itself, cause phase shifts of the eye rhythm (Eskin, 1977; Fig. 3), nor does it prevent the expression of the circadian rhythm (Jacklet, 1973). These results with HMLC indicate that chemical synaptic transmission is probably involved in mediating neural enhancement of resetting.

\section{Dose-response relationship}

In attached eyes, bath application of 5-HT at concentrations of 1-10 $\mu \mathrm{M}$ induced resets that were the same size as resets induced by efferent neural activity. At concentrations of 0.1 and 0.01 $\mu \mathrm{M}$, phase-shifting was unreliable or absent, presumably because these doses were below the pharmacological threshold. At concentrations of 0.6 and $2.0 \mathrm{~mm}$, phase-shifting was also unreliable or absent. In this case the receptors mediating 5-HT action may have been desensitized by saturation (Gerschenfeld and Paupardin-Tritsch, 1974a, b; Gerschenfeld and Stefani, 1966).

Phase-shifting specific to $5-H T$

In contrast to 5-HT, agonists of dopamine, octopamine, and acetylcholine failed to produce phase shifts at a concentration of $10 \mu \mathrm{M}$. Agonists of these transmitters were tried because these transmitters are known to affect the electrophysiological activity of the eye, or to be present in the eye (Corrent and Eskin, 1982; Corrent et al., 1978; Eskin, 1977; Jacklet, 1968; Luborsky-Moore and Jacklet, 1976). Additional putative transmitters have been tried by Eskin (1977) and have failed to cause phase-shifting in eyes pretreated with LD 12/12. In Eskin's tests, however, the putative transmitters were not applied under circumstances in which efferent neural activity has a strong effect on resetting.

\section{Action of exogenous 5-HT not mediated by chemical synapses}

The phase-shifting activity of exogenous 5-HT was not blocked by HMLC. Accordingly, the action of 5-HT is probably not mediated by chemical synaptic transmission. Therefore, 5-HT probably acts directly on the cells that perform the phase shift (the oscillator cells) or on cells that are electrically coupled to 
the oscillator cells. According to recent evidence from the marine snail Bulla gouldiana (Block and McMahon, 1984; Block et al., 1984; McMahon et al., 1984), the oscillator cells are probably the ones that Jacklet and Rolerson (1982; Jacklet, 1976) named "D cells." The possibility that 5-HT acts on cells that are electrically coupled to the oscillator cells should not be rejected out of hand. Gap junctions, dye-coupling and electrical coupling occur extensively among the cells of the retina (Block et al., 1984; Jacklet, 1973; Jacklet and Rolerson, 1982; Jacklet et al., 1982; Strumwasser et al., 1979).

\section{Mimicry of neural action by 5-HT}

We collected four types of data that bear on the question of whether 5-HT mimics neural action. The four types are (1) the action of 5-HT on detached eyes, (2) the combined action of 5-HT and neural activity on attached eyes, (3) the dependence of 5-HT action on the phase of 5-HT application, and (4) the dependence of 5-HT action on duration of light.

1. The action of 5-HT on detached eyes. Corrent, McAdoo, and Eskin (1978) have already shown that 5-HT can reset the eye rhythm. In their experiments, however, there was no basis for comparing the action of 5-HT with the action of the optic efferents. Their duration of light was always $12 \mathrm{hr}$ (the duration of light on the final day of LD), and the maximal reset was only about $3 \mathrm{hr}$. The resetting action of efferent neural activity is greatest when the duration of light is about $21 \mathrm{hr}$, in which case the eye rhythm can be reset by as much as $11 \mathrm{hr}$. The present experiments show that, following a $21 \mathrm{hr}$ day, 5 -HT can produce resets that are as large as those produced by neural activity.

2. The combined action of 5-HT and efferent neural activity. If neural activity and 5-HT were to cause resetting by separate mechanisms, the actions of 5-HT and neural activity should summate. Figure 5 shows, however, that $1-10 \mu \mathrm{m}$ 5-HT induces no resetting in addition to that induced by neural activity itself.

If 5-HT and neural activity use independent mechanisms, then a dose of 5-HT that exceeds the maximum effective dose should not interfere with neural enhancement. The data show, however, that an excessive dose of 5-HT blocks neural enhancement, as would be expected if neural activity and exogenous 5-HT used the same mechanism. Saturation desensitization of 5-HT receptors could explain the ineffectiveness of high doses of 5-HT as well as the blockade of action by serotonergic efferent fibers.

3. Phase-dependence of 5-HT action. Prichard and Lickey (1981b) found that neural activity enhanced resetting only at certain phases of the rhythm. Neural activity during the $3 \mathrm{hr}$ interval of ZT 3-ZT 6 enhanced resetting nearly as well as neural activity of unlimited duration. Neural activity during the $6 \mathrm{hr}$ interval of ZT 21-ZT 3 did not markedly enhance resetting. Prichard and Lickey (1981b) referred to ZT 3-6 as the critical period. (The term critical phase is more apt, and we shall use the latter term from here on.)

In the present studies, the action of 5-HT was also phasedependent (Fig. 6). Serotonin from ZT 23 to 7, an interval that includes Prichard and Lickey's (1981b) critical phase, induced resetting that was identical to that induced by nerve activity. In contrast, 5-HT at ZT 7-15 did not induce resetting; and 5-HT at $\mathrm{ZT} 15-23$ induced submaximal resetting.

Although the effective phase interval for 5-HT action includes the effective phase for neural action, it is uncertain that the effective phases for 5-IIT and neural activity are precisely identical. The uncertainty arises because Prichard and Lickey (1981b) did not explore thoroughly the efficacy of neural activity at all phases of the rhythm and because, in the present study, we used rather long 5-HT pulses. Despite these uncertainties, it is clear that the response of the eye clock to both 5-HT and ncural activity is phase-dependent and that the clock responds identically to both during the critical phase for neural activity.
4. Dependence of resetting magnitude on duration of light. Prichard and Lickey (1981a) showed that the magnitude of the phase shift induced by neural activity was markedly dependent on the duration of light on the final day in LD. If 5-HT and neural activity operate by different mechanisms, it is unlikely that the response to 5-HT would depend on duration of light in the same way. The data, however, show that 5-HT precisely duplicates the neural action. Thereforc, 5-HT and the cfferent axons probably activate the same resetting mechanism.

\section{Cyclic recurrence of sensitivity to $5-H T$}

Serotonin was effective at ZT 23-7 during both the first and the second cycles in vitro. Prichard and Lickey (1981b) did not test the efficacy of neural activity during the second cycle. The present results, therefore, add to previous knowledge by suggesting that the critical phase recurs cyclically in culture.

\section{Comparison with previous work on phase-shifting by 5-HT}

Corrent et al. $(1978,1982)$ described phase shifts in response to 5-HT that are in some ways similar and in other ways different from the phase shifts described here. In their experiments, all Aplysia were entrained to LD 12/12 and the eyes were removed at the end of the light time. The preparation was darkened at the normal time of dusk. Thus, their data are comparable to our data using a light duration of $12 \mathrm{hr}$. They applied 5-HT in $6 \mathrm{hr}$ pulses, beginning at various phases of the rhythm. We used $8 \mathrm{hr}$ pulses, and with a light duration of $12 \mathrm{hr}$, the 5-HT was always applied in the early subjective day. They found that 5-HT, during the early subjective day (ZT 22-4), evoked delaying phase shifts of up to $3 \mathrm{hr}$. We found that 5-HT (ZT 23-7) evoked phase delays of less than $1 \mathrm{hr}$, and these delays were not significantly different from zero (Figs. 2 and 7). Thus, there is a slight discrepancy in the size of the phase shift obtained by the two laboratories when 5-HT was applied near subjective dawn. We feel that the discrepancy is not great, considering the differences in drug-pulse duration and time of drug application.

The effective doses of 5-HT reported here are similar to those reported by Corrent et al. $(1978,1982)$. In both studies, dopamine and acetylcholine agonists did not produce phase-shifting. In both studies, the phase-shifting action of 5-HT was not blocked by HMLC.

The greatest difference between our results and those of Corrent et al. (1978) is in the maximum size of the resetting response. Our maximum reset is approximately $11 \mathrm{hr}$, while theirs is about $3 \mathrm{hr}$. The larger size of our resets is due to the prolonged duration of light on the last light cycle before 5-HT treatment. Since neural enhancement of resetting becomes obvious when light duration increases beyond $12 \mathrm{hr}$, neurally enhanced resetting is probably involved in natural entrainment of the eye clocks by long summer days.

\section{Possible role of $C A M P$}

Eskin et al. (1982) have shown that the phase shift they observed in response to 5-HT applied at ZT 5-11 is mediated by cAMP. We suspect that cAMP also mediates the larger phase shifts induced by 5 -HT following a long day (Jordan et al., 1980). Experiments are needed to clarify the quantitative relationship between the amount of cAMP formed and magnitude of resetting in response to $5-\mathrm{HT}$.

\section{Is 5-HT a clock-regulatory transmitter?}

Prior to this report, the suspicion that 5-HT is a clock-regulating transmitter has been based on three points: 5-HT can reset the eye clock, 5-HT is synthesized in the eye, and 5-HT is contained in the cye (Corrent et al., 1978, 1982). The ocular 5-HT resides in fine neural processes but not in cell bodies or non-neural cells (Goldstein et al., 1984). This paper establishes a fourth point: 
The action of 5-HT mimics the action of efferent neural activity. Thus, 5-HT is now an even stronger contender for designation as a clock-rcgulatory transmitter. This is the first time a putative neurotransmitter has been shown to mimic a neural action on a circadian clock.

It remains to be shown that $5-\mathrm{HT}$ is released by activity of the optic efferents, that the neurites containing 5-HT are the terminals of the efferent axons, and that the efferent axons have an uptake mechanism for 5-HT. These additional points are important, for 5-HT could well mimic the optic efferents without being the efferent transmitter. In Limulus, for example, exogenous serotonin mimics the optic efferents, but the efferent transmitter is almost certainly octopamine (Barlow et al., 1977; Battelle, 1980; Kass and Barlow, 1984).

\section{Circadian function of optic efferents}

What kind of information is transmitted to the eye clock by the optic efferents and their transmitters? Eskin ct al. have speculated that the optic efferents are an entrainment pathway for the eye clock and that 5-HT may be an entrainment transmitter (Corrent and Eskin, 1982; Corrent et al., 1982; Eskin, 1979). Our experiments contain information that is relevant to this spcculation.

When an attached eye is reset by postponing dark onset, the efferent activity might be a zeitgeber that specifies the size of the reset, or it might be an activator that causes the expression of a reset whose size is determined by other mechanisms. Prichard and Lickey (1981c) explored this issue and concluded that efferent neural activity is a resetting activator, not a zeitgeber.

The present results with 5-HT strengthen the interpretation that the optic efferents activate resetting but do not specify the magnitude of resetting. The offset of light (the resetting stimulus) was applied to isolated eyes in vitro. Several hours later, phaseshifting was brought about by applying 5-HT. The size of the phase shift was dependent on the duration of light. Since 5-HT was applied at a constant phase and in a constant dose, we must conclude that the size of the reset was not determined by information in the drug application but by information about the duration of light that was stored within the eye. Thus, in this system, as elsewhere in Aplysia (Kandel and Schwartz, 1982; Mandelbaum et al., 1979; Shimahara and Tauc, 1977; Weiss et al., 1979), 5-HT appears to be a modulatory agent as opposed to an information-bearing agent. It may be more precisely correct to refer to the optic efferents as a clock-regulatory pathway than as a clock-entrainment pathway.

\section{References}

Audesirk, T. (1979) A field study of growth and reproduction in Aplysia californica. Biol. Bull. 157: 407-421.

Barlow, R. B., S. C. Chamberlain, and E. Kaplan (1977) Efferent inputs and serotonin enhance the sensitivity of Limulus eye. Biol. Bull. 153: 414.

Battelle, B.-A. (1980) Neurotransmitter candidate in the visual system of Limulus polyphemus: Synthesis and distribution of octopamine. Vis. Res. 20:911-922.

Block, G. D., and D. G. McMahon (1983) Localized illumination of the Aplysia and Bulla eye reveals new relationships between retinal layers. Brain Res. 265: 134-137.

Block, G. D., and D. G. McMahon (1984) Cellular analysis of the Bulla ocular circadian pacemaker system III: Localization of the circadian pacemaker. J. Comp. Physiol. 155: 387-395.

Block, G. D., and T. L. Page (1979) Effect of efferent activity on entrainment of the Aplysia eye. Comp. Biochem. Physiol. 62A: 635638 .

Block, G. D., D. J. Hudson, and M. E. Lickey (1974) Extraocular photoreceptors can entrain the circadian oscillator in the eye of Aplysia. J. Comp. Physiol. 89: 237-249.

Block, G. D., D. G. McMahon, S. F. Wallace, and W. O. Friesen (1984) Cellular analysis of the Bulla ocular circadian pacemaker system I: A model for retinal organization. J. Comp. Physiol. 155: 365-378.
Cedar, H., and J. Schwartz (1972) Cyclic adenosine monophosphate in the nervous system of Aplysia californica. J. Gen. Physiol. 60:570587.

Corrent, G., and A. Eskin (1982) Transmitterlike action of serotonin in phase shifting a rhythm from the Aplysia eye. Am. J. Physiol. 242: R333-338.

Corrent, G., A. Eskin, and I. Kay (1982) Transmitter like action of serotonin in phase shifting a rhythm from the Aplysia eye. Am. J. Physiol. 242: R333-338.

Corrent, G., D. J. McAdoo, and A. Eskin (1978) Serotonin shifts the phase of the circadian rhythm from the Aplysia eye. Science 202: 977-979.

Eskin, A. (1971) Properties of the Aplysia visual system: In vitro entrainment of the circadian rhythm and centrifugal regulation of the eye. Zeit. vergl. Physiol. 74: 353-371.

Eskin, A. (1977) Neurophysiological mechanisms involved in photoentrainment of the circadian rhythm from the Aplysia eye. J. Neurobiol. 8: 273-299.

Eskin, A. (1979) Circadian system of the Aplysia eye: Properties of the pacemaker and mechanisms of its entrainment. Fed. Proc. 38: 2573-2579.

Eskin, A., and G. Corrent (1977) Effects of divalent cations and metabolic poisons on the circadian rhythm from the Aplysia eye. J. Comp. Physiol. A 117: 1-21.

Eskin, A., C.-Y. Lin, and D. J. McAdoo (1982) Mechanism for shifting the phase of a circadian rhythm by serotonin: Involvement of cAMP. Proc. Natl. Acad. Sci. USA 79: 660-664.

Gerschenfeld, H., and D. Paupardin-Tritsch (1974a) Ionic mechanisms and receptor properties underlying the responses of molluscan neurons to 5-hydroxytryptamine. J. Physiol. (Lond.) 243: 427-456.

Gerschenfeld, H., and D. Paupardin-Tritsch (1974h) On the transmitter function of 5-hydroxytryptamine at excitatory and inhibitory monosynaptic junctions. J. Physiol. (Lond.) 243: 457-481.

Gerschenfeld, H., and E. Stefani (1966) An electrophysiological study of 5-hydroxytryptamine receptors of neurons in the molluscan nervous system. J. Physiol. (Lond.) 185: 684-700.

Goldstein, R., H. B. Kistler, H. W. M. Steinbusch, and J. H. Schwartz (1984) Distribution of serotonin-immunoreactivity in juvenile Aplysia. Neuroscience 11: 535-547.

Halstead, D. C., and J. Jacklet (1974) Effects of calcium and magnesium on facilitation of a unitary synaptic potential in neuron R15 of Aplysia. Comp. Biochem. Physiol. 47 A: 991-1003.

Harf, L., S. Arch, and A. Eskin (1976) Polypeptide secretion from the eye of Aplysia californica. Brain Res. 111:295-299.

Jacklet, J. W. (1968) Synchronized neuronal activity and neurosecretory function of the eye of Aplysia. Proc. Int. Union Physiol. Sci. 7: 213.

Jacklet, J. W. (1969) A circadian rhythm of optic nerve impulses recorded in darkness from the isolated eye of Aplysia. Science 164: 562-564.

Jacklet, J. W. (1973) The circadian rhythm in the eye of Aplysia. Effects of low calcium and high magnesium. J. Comp. Physiol. 87: 329-338.

Jacklet, J. W. (1974) The effects of constant light and light pulses on the circadian rhythm in the eye of Aplysia. J. Comp. Physiol. 90: 3345.

Jacklet, J. W. (1976) Dye marking in the eye of Aplysia. Comp. Biochem. Physiol. 55A: 373-377.

Jacklet, J. W., and C. Rolerson (1982) Electrical activity and structure of retinal cells of the Aplysia eye: II. Photoreceptors. J. Exp. Biol. 99; 381-395.

Jacklet, J. W., L. Schuster, and C. Rolerson (1982) Electrical activity and structure of retina cells of the Aplysia eye: I. Secondary neurons. J. Exp. Biol. 99: 369-380.

Jordan, W. P., S. O. Hiaasen, and M. E. Lickey (1980) A cAMP analog with a phosphodiesterase inhibitor resets the phase of the circadian oscillator in the Aplysia eye. Soc. Neurosci. Abstr. 6: 833 .

Kandel, E. R., and J. Schwartz (1982) Molecular biology of learning: modulation of transmitter release. Science 218: 433-443.

Kass, L., and R. B. Barlow (1984) Efferent neurotransmission of circadian rhythms in Limulus lateral eye. J. Neurosci. 4: 903-917.

Luborsky-Moore, and J. W. Jacklet (1976) Aplysia eye: Modulation by efferent activity. Brain Res. 115: 501-505.

McMahon, D. G., S. F. Wallace, and G. D. Block (1984) Cellular analysis of the Bulla pacemaker system II: Neurophysiological basis of circadian rhythmicity. J. Comp. Physiol. 155: 379-386.

Mandelbaum, J., M. Koester, M. Schonberg, and K. R. Weiss (1979) 
Cyclic AMP mediation of the excitatory effect of serotonin in the heart of Aplysia. Brain Res. 177: 388-394.

Nadakavukaren, J. J. (1980) Pharmacological properties of a phaseresetting pathway in the eye-brain circadian system of Aplysia californica. Doctoral dissertation, University of Oregon, Eugene, OR.

Prichard, R. G., and M. E. Lickey (1981a) In vitro resetting of the circadian clock in the Aplysia eye with LL to DD transitions. I. Importance of efferent activity in optic nerve. J. Neurosci. 1: 835-839.

Prichard, R. G., and M. E. Lickey (1981b) In vitro resetting of the circadian clock in the Aplysia eye with LL to DD transitions. II. The critical period for optic nerve activity. J. Neurosci. 1: 840-845.

Prichard, R. G., and M. E. Lickey (1981c) In vitro resetting of the circadian clock in the Aplysia eye with LL to DD transitions. III. Location of photoreceptors that signal LL/DD. J. Neurosci. I: 846848.
Shimahara, T., and L. Tauc (1977) Cyclic AMP induced by serotonin modulates activity of an identified synapse in Aplysia by facilitating the active permeability to calcium. Brain Res. 127: 168-172.

Strumwasser, F., R. Alvarez, D. P. Viele, and J. C. Woolum (1979) Structure and function of a neuronal circadian oscillator system. In Biological Rhythms and their Central Mechanisms, M. Suda, O. Hayaishi, and H. Nakagawa, eds., pp. 41-56, Elsevier/North Holland, Amsterdam.

Weiss, K. R., D. E. Mandelbaum, M. Schonberg, and I. Kupfermann (1979) Modulation of buccal muscle contractility by serotonergic metacerebral cells in Aplysia: Evidence for a role of cyclic adenosine monophosphate. J. Neurophysiol. 42: 791-803. 